DOI: https://doi.org/10.14232/actahisp.2020.25.9-22

\title{
DIPLOMACIA DE CUMBRES A NIVEL INTERREGIONAL: UN ANÁLISIS DESDE LA RELACIÓN ENTRE LA UNIÓN EUROPEA Y LATINOAMÉRICA
}

\author{
Lizeth VANessa Ayala Castiblanco
}

\author{
Universidad Corvinus de Budapest
}

\begin{abstract}
Resumen: La diplomacia de cumbres es una práctica cada vez más común, aunque poco estudiada, en las relaciones internacionales. Los encuentros periódicos entre Jefes de Estado y de Gobierno se constituyen como un escenario desde el cual se toman importantes decisiones para afrontar los crecientes retos comunes a nivel internacional. Esta práctica diplomática no solo ha fortalecido las relaciones entre Estados sino que también ha favorecido el desarrollo de relaciones interregionales cuya importancia sobresale en un contexto de latentes amenazas transnacionales. El objetivo de este artículo es presentar el marco conceptual desde el cual se estudia la diplomacia de cumbres y analizar el caso particular de la relación birregional entre la Unión Europea y Latinoamérica cuyos avances más significativos se han realizado en el contexto de esta práctica diplomática. Dentro del análisis, el seguimiento a las declaraciones conjuntas permite formular un diagnóstico acerca de los valores comunes y las áreas de cooperación que ambas regiones han priorizado a lo largo de sus veinte años de asociación estratégica. Temas como la igualdad de género y el desarrollo sostenible han cobrado cada vez mayor relevancia en la agenda birregional en consonancia con las tendencias internacionales. Sin embargo, más allá de las declaraciones conjuntas, es necesario crear mecanismos de seguimiento a los acuerdos alcanzados con el fin de fomentar la transparencia en los proyectos conjuntos y poder determinar en qué medida la diplomacia de cumbres cumple con su objetivo de fortalecer las relaciones entre actores a nivel internacional.
\end{abstract}

Palabras clave: diplomacia de cumbres, interregionalismo, Latinoamérica, Unión Europea.

\begin{abstract}
Summit diplomacy is an increasingly common but overlooked practice in international relations. Periodic meetings between Heads of State and Government constitute a space from which important decisions are made in order to face growing common challenges at the international level. This diplomatic practice has not only strengthened relations between states but has also favored the development of interregional relations which are important in the context of latent transnational threats. This paper aims to present the conceptual framework for the study of summit diplomacy and to analyze the particular case of the biregional relationship between the European Union and Latin America, whose most significant advances were achieved in the context of this diplomatic practice. Within the analysis, joint declarations are examined in order to provide a diagnosis about the common values and the areas of cooperation that both regions have prioritized throughout their twenty years of strategic partnership. Issues such as gender equality and sustainable development have become increasingly relevant on the biregional agenda in line with international trends. However, beyond joint declarations, it is necessary to create follow-up mechanisms to the reached agreements in order to promote the transparency of joint projects and to be able to determine to what extent summit diplomacy fulfills its objective of strengthening relations between actors at the international level.
\end{abstract}

Keywords: Summit Diplomacy, Interregionalism, Latin America, European Union.

Acta Hispanica 25: 9-22, 2020, ISSN 1416-7263, e-ISSN 2676-9719 | 9 
Diplomacia de cumbres a nivel interregional: un análisis desde la relación entre la Unión Europea y Latinoamérica

\section{Introducción}

En un contexto de crisis globales a nivel económico, político, social e incluso a nivel sanitario, se ha puesto en evidencia el hecho de que la acción unilateral es insuficiente para afrontar los retos comunes que surgen en el entorno internacional. La cooperación, no solo entre Estados sino también entre otros actores internacionales, surge como una herramienta indispensable para hacer frente a estos retos mancomunados en el sistema internacional. Así, las relaciones bilaterales, birregionales o multilaterales son esfuerzos encaminados hacia la búsqueda de sinergias para afrontar problemáticas en común.

La diplomacia de cumbres es una de las manifestaciones más importantes de las relaciones entre actores internacionales. Frecuentemente se constituye como el principal canal de diálogo entre las partes y el escenario en el que se gestan múltiples iniciativas conjuntas. Además, es el espacio en el cual se establece un mayor nivel de confianza entre los líderes -al facilitar el contacto personal más allá de los eventos oficialesposibilitando la creación de proyectos de cooperación y el establecimiento de posiciones comunes en foros multilaterales.

Sin embargo, el desarrollo de esta práctica diplomática supone también una serie de esfuerzos reflejados principalmente en su compleja organización logística, lo que genera un cuestionamiento acerca de la necesidad de realizar estos eventos considerando sus potenciales beneficios y contrastándolos con sus costos. Más aún, la proliferación de cumbres en torno a distintos temas de la agenda internacional en los últimos años ha suscitado un debate sobre la verdadera contribución de algunas de ellas. Una evaluación de los beneficios y desventajas esta práctica diplomática puede ser desarrollada con mayor efectividad partiendo del análisis de un caso específico.

En este contexto, la relación birregional entre la Unión Europea y América Latina es un caso de estudio de particular relevancia en el ámbito de la diplomacia de cumbres. Estas dos regiones mantienen un diálogo político regular desde hace veinte años cuya máxima expresión es la realización de cumbres bienales. Desde 1999, la Unión Europea y Latinoamérica acordaron la creación de una asociación estratégica birregional basada en sus fuertes lazos culturales y su adherencia a una serie de valores y posiciones comunes derivados de su historia compartida. Las cumbres bienales se han constituido como la única oportunidad de reunión de todos los líderes nacionales de ambas regiones y el espacio desde el cual se toman las decisiones más importantes en materia birregional.

En este artículo se abordará, en primer lugar, el marco conceptual que rodea los estudios sobre la diplomacia de cumbres, poniendo de manifiesto la importancia de la misma, sus retos principales y las críticas que ha recibido como práctica diplomática. Posteriormente, se analizará el caso de la relación birregional entre la Unión Europea y Latinoamérica resaltando sus antecedentes, hitos principales y su situación actual haciendo énfasis en el desarrollo del proceso de cumbres bienales. Luego se examinará 
el diálogo político entre estas regiones, manifestado a través de las declaraciones conjuntas, revisando los planteamientos más importantes de dichos documentos. Finalmente, se presentarán las conclusiones principales a partir del análisis del marco conceptual y el caso estudiado.

\section{La diplomacia de cumbres desde un marco conceptual}

La diplomacia de cumbres, aunque frecuentemente cuestionada, se constituye como un desarrollo irreversible en la diplomacia moderna (Melissen, 2003). Los líderes políticos están cada vez más interesados en un manejo propio de la política exterior por lo que se han involucrado con mayor frecuencia en el diálogo directo con sus pares a nivel internacional. La necesidad de afrontar retos comunes desde una posición conjunta y la posibilidad de crear una red de relaciones que sirvan a los intereses nacionales son incentivos para la organización de este tipo de encuentros (Freres, 2004).

Históricamente, las cumbres solo se convocaban ante la urgencia de un asunto importante por resolver (Freres, 2004). Con el paso del tiempo y el aumento en las facilidades del transporte internacional, las reuniones entre líderes se hicieron más frecuentes. En la actualidad, en un contexto internacional que es cada vez más complejo, la diplomacia de cumbres se constituye como un mecanismo de gestión de la interdependencia realizada principalmente desde las iniciativas multilaterales (Freres, 2004). En este sentido, según Rojas Aravena (2009), los encuentros periódicos entre Jefes de Estado y de Gobierno se constituyen como una expresión insigne del multilateralismo en tanto definen la agenda subregional y, a su vez, plantean acuerdos cruciales en materia internacional. Las cumbres se constituyen como un diálogo directo, periódico y "rutinizado" al más alto nivel, lo que favorece el desarrollo de iniciativas conjuntas y permite darle continuidad y seguimiento a los proyectos en curso (Rojas Aravena 2009).

Sin embargo, la preparación de este tipo de eventos requiere de grandes esfuerzos tanto por parte de los organizadores como de los participantes. La dificultad para conciliar las agendas de los líderes, los altos costos en términos monetarios, la logística requerida para organizar las reuniones, entre otros, constituyen importantes retos a la hora de planear y llevar a cabo una cumbre bien sea bilateral, birregional o multilateral. Por otro lado, es importante resaltar el carácter mediático de las cumbres. Su impacto en los medios puede llegar a ser bastante significativo para los líderes participantes ya que las cumbres se convierten en una vitrina para las audiencias nacionales e internacionales que hacen seguimiento a las acciones y decisiones de sus líderes en este tipo de eventos. En este contexto, la realización de este tipo de eventos tiene también una carga simbólica considerando nivel decisorio que ostentan sus interlocutores (Taiana, 2009).

La importancia de la diplomacia de cumbres para las relaciones internacionales se basa en su protagonismo como canal de comunicación entre las partes. Las cumbres 
Diplomacia de cumbres a nivel interregional: un análisis desde la relación entre la Unión Europea y Latinoamérica

acercan a los líderes nacionales y regionales, los convocan en un mismo espacio y les dan la posibilidad de concertar nuevas iniciativas y hacer seguimiento a los proyectos conjuntos en curso. Inclusive, en algunas ocasiones, los beneficios de las cumbres van más allá de sus propósitos específicos pues también se puede aprovechar la presencia de los mandatarios para generar espacios de diálogo adicionales a los previstos en la agenda oficial (Taiana, 2009). En el caso particular de las relaciones interregionales, el intercambio y el diálogo al más alto nivel político son decisivos para el fortalecimiento de los vínculos establecidos por lo que, sin importar su forma y propósito, "el interregionalismo puede entenderse, al menos en parte, como un ejercicio de cumbres" (Gardini - Malamud, 2016: 10).

Sin embargo, pese a los posibles beneficios y resultados positivos que podrían derivarse de la celebración de cumbres -y aunque la falta de ellas pueda considerarse como un signo de estancamiento en una relación bilateral- la diplomacia basada en cumbres también ha sido analizada desde una mirada crítica y escéptica. Por ejemplo, Dunn (1996) ha cuestionado hasta qué punto algunas de las cumbres se convierten en meras reuniones simbólicas sin contenido sustancial de fondo o avances concretos. En algunos casos, la carencia de mecanismos de seguimiento a los planteamientos surgidos en las cumbres hace que sea difícil distinguir las cumbres verdaderamente significativas de las que no lo son.

En este sentido, cabe anotar que la proliferación de compromisos internacionales de esta naturaleza ha aumentado exponencialmente en los últimos años. Y no solo se trata de cumbres bilaterales o birregionales. También se organizan foros internacionales concernientes a la discusión de problemas en áreas como el cambio climático, el desarrollo sostenible, la lucha contra las drogas, el crimen transnacional, entre otros temas. Por tanto, considerando que la organización de las cumbres requiere de grandes esfuerzos logísticos, las cumbres "tienen que competir por los recursos humanos y financieros contra un gran y creciente número de otros compromisos internacionales, incluso regionales, y nacionales" (Gardini - Malamud, 2016: 11).

Más aún, la saturación de cumbres internacionales ha llegado al punto en que incluso se superponen unas a otras en la agenda internacional. En este contexto, Gardini y Malamud (2016) señalan que cuando el ejercicio de cumbres se torna excesivo llega a un punto de agotamiento máximo. Por tanto, “independientemente del interés, el valor y los resultados de cada uno de estos eventos, su proliferación tiende a disminuir los rendimientos marginales para todos los interesados" (Gardini - Malamud, 2016: 10)ํ. Además, se realiza tal cantidad de cumbres que eventualmente "es difícil señalar alguna como especialmente significativa" (Freres, 2004: 112).

No obstante, aunque la diplomacia de cumbres suele ser criticada por sus altos costos en términos de logística en comparación con los beneficios concretos que puede generar, con frecuencia gran parte de su verdadero valor reside en los encuentros que

${ }^{1}$ Todas las traducciones han sido realizadas por la autora. 
no llegan a ser de conocimiento público. Más aún, uno de los aportes más importantes de las cumbres es el fortalecimiento de la comunicación política y la confianza al más alto nivel (Yepe, 2011). De hecho, las cumbres "suelen propiciar el impulso decisivo para hacer avanzar acuerdos y proyectos que, a niveles inferiores de la burocracia gubernamental, difícilmente podrían despegar" (Yepe, 2011: 249). Con frecuencia, estos beneficios de las cumbres tienden a ser desestimados al ser difíciles de identificar en algunos casos.

Los acuerdos alcanzados en las cumbres suelen ser presentados en las declaraciones oficiales que se emiten tras la culminación de estos eventos. En este sentido, es importante reconocer el esfuerzo diplomático que tiene como resultado la publicación de una declaración. Como lo señala Ferrero-Waldner (2009: 55), “detrás de cada declaración de Jefes de Estado y de Gobierno hay un profundo proceso de trabajo y diálogo para aunar puntos de vista y posiciones en temas considerados estratégicos para las dos regiones". En las cumbres se adquieren compromisos, se reafirman valores compartidos, "se toman decisiones en cuanto al lanzamiento de procesos de negociación y se decide la puesta en marcha de importantes programas de cooperación" (Ferrero-Waldner, 2009: 56).

Generalmente, las declaraciones finales de las cumbres son preparadas por los equipos técnicos buscando reflejar los consensos alcanzados (Rojas Aravena, 2009). Sin embargo, muchas de estas declaraciones se caracterizan por su multiplicidad de acuerdos y poco nivel de compromiso y cumplimiento real de los mismos (Rojas Aravena, 2009). Las declaraciones suelen abordar muchos y diversos temas, lo que a veces resulta en bajos niveles de articulación y coordinación de políticas a posteriori. La excesiva multiplicidad de compromisos -cuyo cumplimiento es a veces ambiguo- es un error recurrente en las declaraciones conjuntas, y en la diplomacia de cumbres en general.

Estos cuestionamientos sobre la forma y fondo de las declaraciones que surgen como resultado de las cumbres requiere del análisis de un caso de estudio en particular que permita poner a prueba este tipo de planteamientos y revelar la verdadera importancia de las cumbres y de las decisiones allí tomadas. A continuación se analizará el caso de la relación birregional entre la Unión Europea y Latinoamérica con el objetivo de revisar el desarrollo de la diplomacia de cumbres y sus resultados en un contexto específico.

\section{La relación birregional Unión Europea - Latinoamérica como caso de estudio}

La relación birregional entre la Unión Europea y Latinoamérica se cimienta en una trayectoria histórica compartida desde la época de la colonización que tuvo como resultado la creación de lazos estrechos de un lado y otro del Atlántico (Grabendorff, 2003). En este sentido, la herencia cultural europea ha sido un componente de gran importancia para la construcción de la identidad latinoamericana. Del mismo modo, el 
Diplomacia de cumbres a nivel interregional: un análisis desde la relación entre la Unión Europea y Latinoamérica

discurso europeo hacia América Latina tiende a resaltar estos vínculos compartidos como base de su aproximación a la región. Sin embargo, la relación no siempre ha recibido especial atención por parte de ambas regiones por lo que su continuidad se ha mantenido inconstante.

Las décadas posteriores a la independencia de las naciones latinoamericanas constituyeron un periodo de transición en la relación birregional. Uno de los cambios principales fue el retiro de España y Portugal como mediadores del flujo comercial entre Europa y Latinoamérica. Como consecuencia, se estableció una relación comercial directa con los demás países europeos basada en la exportación de materias primas. Gran Bretaña, Francia y Alemania se convirtieron en los principales socios comerciales de los países latinoamericanos recién independizados. A medida que aumentaban los intercambios comerciales, surgió una nueva dependencia económica de Europa. Sin embargo, a fines del siglo XIX, Estados Unidos emergió como un actor económico de mayor importancia para los países latinoamericanos. Tras el auge industrial y económico estadounidense, el siglo XX estuvo marcado por el rol protagónico de Estados Unidos en América Latina. Sin embargo, cabe destacar que esta tendencia tuvo un alcance diferenciado en los países latinoamericanos, ya que Chile, Argentina, Uruguay y, en parte, Brasil mantuvieron una fuerte orientación comercial hacia Europa.

Posteriormente, y tras el fin de la Segunda Guerra Mundial, la emergencia del proceso de integración europeo generó un cambio en la manera en que Europa se relacionaba con el mundo. De hecho, la creación de la Comunidad Económica Europea (CEE) marcó el surgimiento de Europa como un actor importante en el sistema internacional (Bretherton - Vogler, 1999). La institucionalización del proceso de integración europea tuvo implicaciones no solo en la percepción propia de la región sino también en su relación con otros actores. Incluso, la CEE se convirtió en un modelo a seguir para América Latina en tanto surgieron diversas iniciativas regionales como el Mercado Común Centroamericano (MCCA) y la Asociación Latinoamericana de Libre Comercio (ALALC) con el objetivo de imitar el proceso europeo.

En la década de 1980 inició un acercamiento birregional cuando la CEE participó en el programa de cooperación para poner fin al conflicto centroamericano respaldando el proceso de paz en Nicaragua, El Salvador y Guatemala (Ayuso - Caballero, 2018). Este fue considerado como uno de los mayores esfuerzos para lograr la estabilidad regional y consolidar la democracia en el continente. El proceso condujo a la creación del Diálogo de San José, que fue la primera iniciativa de entendimiento político birregional. Este tipo de intervención fue visto como un signo del poder normativo europeo emergente en América Latina (Ayuso, 2019).

España y Portugal tuvieron una influencia significativa para impulsar el acercamiento euro-latinoamericano en los años siguientes. Desde su ingreso a la CEE en 1986, estos países se convirtieron en el puente entre ambas regiones debido a sus estrechos vínculos y afinidad cultural con América Latina (Ayuso - Gratius, 2016). Los 
lazos existentes entre estos dos países europeos y América Latina cimentaron la noción de "Iberoamérica" como una zona de importantes intercambios a nivel social y cultural.

En la década de los noventa, la relación birregional adquirió especial relevancia debido a cambios importantes en ambas regiones. Por un lado, luego del período conocido como "la década perdida" en América Latina, se desencadenó una transformación hacia políticas de apertura liberal en la región. Las nuevas reformas económicas facilitaron los flujos de inversión en procesos de privatización de empresas estatales, lo que aumentó el interés europeo en la región (Grabendorff, 2004). Además, América Latina consideró a la recién creada Unión Europea (UE) como un aliado para reducir la dependencia económica que había generado hacia Estados Unidos. Por otro lado, la creación de la UE y el desarrollo de su política exterior jugaron un papel fundamental para repensar el acercamiento europeo hacia otras regiones. En este contexto, la UE planteó un enfoque más institucionalizado hacia América Latina (Freres - Sanahuja, 2005) que se reflejó en el establecimiento de una asociación estratégica entre ambas regiones.

En 1999 se celebró la Primera Cumbre entre Jefes de Estado y de Gobierno de la UE y de América Latina en Río de Janeiro. Durante esta cumbre se hizo el lanzamiento oficial de la asociación estratégica birregional en un entorno de altas expectativas. El objetivo principal era establecer una serie de programas de acción conjunta para fortalecer la relación entre ambas regiones. Así, la asociación estratégica se basa en tres pilares principales: diálogo político, cooperación regional y comercio (Cervantes, 2000). Desde entonces, y cada dos años, se han realizado cumbres birregionales tanto en Europa como en América Latina -a excepción del último quinquenio, lo cual se explicará más adelante. En dichas reuniones bienales se toman las decisiones principales sobre nuevos avances en la relación. En este sentido, la diplomacia de cumbres se ha constituido como el principal canal de diálogo político entre ambas regiones. A continuación se enumeran las cumbres bienales que se han celebrado entre la UE y América Latina.

\begin{tabular}{|c|c|c|}
\hline Cumbre & Lugar & Fecha \\
\hline I Cumbre Unión Europea - América Latina & Rio de Janeiro, Brasil & Jun 28-29, 1999 \\
\hline II Cumbre Unión Europea - América Latina & Madrid, España & May 17-20, 2002 \\
\hline III Cumbre Unión Europea - América Latina & Guadalajara, México & May 28-29, 2004 \\
\hline IV Cumbre Unión Europea - América Latina & Viena, Austria & May 12-13, 2006 \\
\hline V Cumbre Unión Europea - América Latina & Lima, Perú & May 16-17, 2008 \\
\hline VI Cumbre Unión Europea - América Latina & Madrid, España & May 17-20, 2010 \\
\hline $\begin{array}{c}\text { VII Cumbre Unión Europea - América Latina } \\
\text { (denominada I Cumbre UE-CELAC) }\end{array}$ & Santiago, Chile & Ene 26-27, 2013 \\
\hline $\begin{array}{c}\text { VIII Cumbre Unión Europea - América Latina } \\
\text { (denominada II Cumbre UE-CELAC) }\end{array}$ & Bruselas, Bélgica & Jun 10-11,2015 \\
\hline
\end{tabular}

Fuente: Elaboración propia con base en Quevedo Flores (2017) 
Diplomacia de cumbres a nivel interregional: un análisis desde la relación entre la Unión Europea y Latinoamérica

A partir de estos encuentros, se han creado una serie de programas de cooperación en áreas específicas de acuerdo a las prioridades establecidas por los líderes de ambas regiones en cada cumbre. Dentro de estos programas se destacan las acciones de Eurosocial -en la reducción de la pobreza y la desigualdad-, Euroclima -en la mitigación de los efectos del cambio climático-, AL-Invest -para incentivar las inversiones y el crecimiento de las PYMES-, Copolad -para el combate al tráfico de drogas- y El Pacto -para la lucha contra el crimen transnacional organizado- entre otros. Además, en 2010 se acordó la creación de la Fundación EU-LAC, actualmente reconocida con el estatus de organización internacional, cuya misión es involucrar a diversos actores de la sociedad civil en el desarrollo de los compromisos birregionales que se acuerdan en los diálogos de alto nivel.

Un obstáculo recurrente a lo largo de los años fue la falta de una institución regional que representara a América Latina en su conjunto ante a la UE. La divergencia de intereses y posturas entre los países de la región impedía lograr un consenso previo a las cumbres birregionales que facilitara la creación de acuerdos de cooperación con la UE y, sobre todo, la liberalización del comercio interregional. Ante este panorama, la UE empezó a entablar negociaciones bilaterales con países a nivel individual y con distintos bloques subregionales como Mercosur y la Comunidad Andina con el fin de avanzar en el plano comercial por esta vía. Esta estrategia ha socavado el papel del diálogo bloque a bloque pero le ha permitido a la UE llegar a acuerdos que no había podido negociar con Latinoamérica en su conjunto. Así, la relación birregional ha estado marcada por el diálogo entre la UE, como actor único, y una plétora de esquemas subregionales que representaban a distintos grupos de países en Latinoamérica.

En el 2011 se inició una nueva etapa en la relación birregional con la creación de la Comunidad de Estados Latinoamericanos y Caribeños (CELAC), el primer bloque regional que agrupó a todos los países de Latinoamérica. CELAC fue reconocida como interlocutora oficial de la región, lo que facilitaría la relación con otros actores internacionales como la UE. Sin embargo, la existencia de los bloques subregionales en Latinoamérica y su negociación paralela con la UE había moldeado la relación birregional a tal punto que CELAC no logró constituirse como la voz única latinoamericana. Por tanto, a pesar de que la creación de CELAC fue el intento de construir una posición común entre todos los países latinoamericanos, un entendimiento en América Latina sigue siendo un objetivo no logrado.

En la actualidad, la relación entre la UE y Latinoamérica está atravesando por uno de los momentos más críticos. Fenómenos como la crisis financiera en 2008 y la crisis migratoria en 2015 impactaron negativamente las relaciones exteriores de la UE (Ayuso, 2019). Además, el proceso del Brexit ha demandado bastante atención en la región y la ha llevado a tomar una actitud más introspectiva (Mori, 2018; Bouzas, 2019). Algunas de las consecuencias de esta situación son la disminución de fondos de cooperación para América Latina, la falta de nuevos proyectos conjuntos y, en general, el mayor desinterés de Europa hacia la relación birregional priorizando cuestiones de su propio 
vecindario. En este contexto, otras regiones han adquirido mayor relevancia -como por ejemplo Medio Oriente- mientras que América Latina ha quedado al margen de la política exterior europea.

Por parte de Latinoamérica, el bajo nivel de institucionalización de CELAC, su falta de consenso, las divisiones ideológicas en torno a la solución de la crisis venezolana y el predominio de los intereses nacionales sobre los regionales han perjudicado la relación con la UE. Además, China ha empezado a tener un papel más importante en la región debido al aumento de los intercambios comerciales y las inversiones, lo que ha disminuido la importancia de otros socios externos para Latinoamérica.

Como consecuencia de los cambios internos tanto en la UE como en América Latina, la relación entró en una fase de estancamiento cuyo síntoma más evidente es la falta de cumbres birregionales desde el año 2015, lo que se constituye como un hecho sin precedentes en la relación. En 2017 se esperaba la realización de la novena cumbre birregional en la ciudad de San Salvador, considerando que El Salvador ostentaba la presidencia pro-témpore de la CELAC. Sin embargo, esta cumbre no se llevó a cabo. Aunque se han realizado un par de reuniones ministeriales en los últimos años, éstas no tienen la misma relevancia e impacto en la toma de decisiones que las cumbres bienales que reúnen a todos los Jefes de Estado y de Gobierno de ambas regiones. Esta situación es interpretada como una fase de estancamiento en la relación en tanto la falta de diálogo imposibilita la creación de nuevos acuerdos birregionales y dificulta la continuidad de los proyectos en marcha.

A pesar de estas circunstancias adversas, la asociación estratégica birregional continúa vigente y algunos de los proyectos de cooperación siguen activos hasta el momento. Varios de estos proyectos conjuntos involucran tanto a actores gubernamentales como actores no estatales, lo que hace que esta relación se constituya como un caso de interregionalismo complejo y polimórfico (Ayuso - Gardini, 2018). Así pues, a pesar de que el espacio de encuentro más importante de la asociación birregional son las cumbres bienales, existe también una compleja red de acuerdos y proyectos de cooperación en torno a los cuales continúa evolucionando la relación.

\section{El diálogo interregional como producto de la diplomacia de cumbres: Una mirada a las declaraciones conjuntas}

Ante el estancamiento del diálogo político birregional por la falta de cumbres entre la UE y Latinoamérica, cabe examinar los resultados concretos de las cumbres realizadas previamente. Una revisión histórica de estos eventos pone en evidencia que los acuerdos alcanzados se plasman principalmente en las declaraciones de las cumbres realizadas. En total se han realizado ocho cumbres birregionales entre la Unión Europea y América Latina (1999, 2002, 2004, 2006, 2008, 2010, 2013, 2015) a lo largo de sus veinte años de asociación estratégica. Un análisis detallado de las declaraciones 
Diplomacia de cumbres a nivel interregional: un análisis desde la relación entre la Unión Europea y Latinoamérica

de estas cumbres permite identificar una serie de patrones alusivos a la evolución misma de la relación birregional.

Las primeras cumbres se caracterizaron por la multiplicidad de compromisos adquiridos en diferentes áreas de acción conjunta. La animosidad por abarcar tantos aspectos de la relación era una muestra de la voluntad política que se evidenciaba en los inicios de la relación. Sin embargo, el planteamiento de numerosos compromisos fue cuestionado a lo largo del tiempo por carecer de un verdadero impacto en la realidad regional más allá de las declaraciones (Grabendorff, 2003; Freres, 2004; Sanahuja, 2004; Malamud, 2010). Esta multiplicidad de planteamientos en las declaraciones fue modificada posteriormente, lo que se vio reflejado en la disminución de la cantidad de propuestas planteadas en estos documentos.

El apoyo al sistema multilateral con base en la Organización de las Naciones Unidas es una de las temáticas más recurrentes en las declaraciones conjuntas. Muchos de los enunciados planteados en estos documentos hacen referencia al compromiso de la asociación estratégica birregional con los principios manifestados en la Carta de las Naciones Unidas. De igual manera, las referencias al derecho internacional y su cumplimiento irrestricto son recurrentes en estos textos, lo cual pone en evidencia el nivel de importancia que tienen las instancias internacionales para la relación birregional entre la UE y Latinoamérica. Además, en varias de las declaraciones se menciona la necesidad de reformar el sistema de Naciones Unidas para hacerlo más inclusivo y más acorde a la realidad internacional actual.

En términos generales, el apoyo al multilateralismo es constante en los textos de las declaraciones y se ve reflejado en la mención de distintas iniciativas multilaterales vigentes en el momento de cada cumbre y la insistencia acerca de la importancia de participar en estas iniciativas. Más aún, muchos de los compromisos planteados en las declaraciones birregionales están inspirados en propuestas realizadas en otras instancias multilaterales donde los países europeos y latinoamericanos han participado. La mención a acuerdos multilaterales como el Protocolo de Kyoto, la Ronda de Doha, el Estatuto de Roma, entre otros, es bastante recurrente.

Otro elemento destacado por su continua aparición en la retórica de las cumbres es la primacía de los llamados valores compartidos que se constituyen como base y punto de partida de la relación birregional. La defensa de la democracia y los derechos humanos se enuncia recurrentemente en todas y cada una de las declaraciones conjuntas emitidas. Sin embargo, la interpretación y práctica de estos valores compartidos puede diferir en ambas regiones de acuerdo a la posición ideológica de los gobiernos en turno. En la actualidad es posible observar que, pese a lo que expresan en las cumbres birregionales, no en todos los países de Latinoamérica ni de la UE se cumple a cabalidad con el respeto a los valores compartidos de protección a la democracia, el Estado de Derecho y los derechos humanos que se proclama en las declaraciones conjuntas. 
En cuanto a la agenda birregional, durante las primeras cumbres se otorgó un énfasis especial a los temas comerciales y el avance de acuerdos económicos entre distintos países y subregiones de Latinoamérica con la UE. Este tema no pudo ser negociado bloque-a-bloque debido a la falta de coordinación entre los países latinoamericanos para entablar una negociación conjunta ante la UE. La lucha contra el terrorismo fue también un tema frecuente en las declaraciones tras los atentados del 11 de septiembre en EEUU, demostrando una alineación con las prioridades de la agenda internacional en aquel entonces. De igual manera, tras la crisis económica del 2008, el apoyo birregional a la modificación de la arquitectura financiera internacional fue un tema recurrente en los textos ante la necesidad de evitar que un colapso financiero similar se presentara nuevamente en el futuro.

Con el paso del tiempo, la agenda birregional ha avanzado hacia la inclusión de nuevos temas y se han identificado otro tipo de prioridades en consonancia con los nuevos retos transnacionales y las necesidades de diferentes actores de la sociedad civil en estas regiones. En este sentido, asuntos como la igualdad de género, el desarrollo sustentable y la seguridad ciudadana, entre otros, han ganado protagonismo en la relación birregional lo que se ha expresado en acuerdos específicos en estos temas. Sin embargo, ante la multiplicidad de temas y compromisos en las declaraciones birregionales, hace falta identificar un mecanismo efectivo de seguimiento tanto a los acuerdos establecidos en años anteriores como a los últimos compromisos, favoreciendo la transparencia de estos procesos pues la mera declaración de intenciones no basta para poder medir el impacto real de la diplomacia de cumbres no solo en la relación birregional entre la UE y Latinoamérica, sino también a nivel internacional.

\section{Conclusión}

La diplomacia de cumbres ha adquirido un rol cada vez más preponderante en la escena internacional. Los beneficios que de ella se derivan están relacionados con la importancia del encuentro personal entre líderes, los acuerdos alcanzados a partir del diálogo político, e incluso, los compromisos que se logran más allá de la agenda formal de estos eventos. Sin embargo, esta práctica no escapa al examen crítico de su desarrollo. La excesiva organización de cumbres ha puesto en cuestión la relevancia de algunas de ellas y ha causado una saturación de la agenda internacional hasta el punto en que, frecuentemente, varios de estos eventos se superponen entre sí.

Si bien, la organización y realización de cumbres se constituye como un ejercicio que supone la inversión de recursos que en algunos casos se consideran excesivos -en comparación con los beneficios tangibles que se obtienen a cambio-; se trata de una práctica que aún es necesaria para el avance de las relaciones internacionales bien sea a nivel bilateral, birregional o multilateral. En el caso concreto de la relación birregional entre la Unión Europea y Latinoamérica, la diplomacia de cumbres es el espacio más importante de toma de decisiones y de encuentro de líderes que, en otras circunstancias, 
Diplomacia de cumbres a nivel interregional: un análisis desde la relación entre la Unión Europea y Latinoamérica

difícilmente se reunirían. En este sentido, la falta de cumbres bienales en los últimos años ha tenido una repercusión bastante negativa para el avance de la relación birregional en tanto ha imposibilitado la creación de nuevos proyectos conjuntos y la coordinación de posiciones comunes en foros internacionales.

El análisis de las declaraciones conjuntas emitidas a lo largo de los veinte años de asociación estratégica revela que los objetivos y áreas de interés entre ambas regiones se han transformado desde un planteamiento vago de principios generales hasta el establecimiento de compromisos concretos en términos de cooperación. En efecto, las declaraciones emitidas durante las primeras cumbres birregionales enunciaban una serie de posiciones comunes en torno a numerosos temas pero se quedaban cortas en cuanto a las acciones a emprender para abordar los problemas mencionados. A lo largo del tiempo, el diálogo birregional evolucionó hacia una serie de compromisos más específicos que se vieron reflejados en la adopción de Planes de Acción desde el año 2010. Además, los temas de la agenda birregional se transformaron desde un enfoque especializado en el aspecto económico y securitario, hacia un mayor interés en temáticas como la acción contra el cambio climático y la igualdad de género, lo que evidencia una transformación positiva de la agenda en correspondencia con las tendencias internacionales.

Adicionalmente, el análisis de las declaraciones conjuntas revela que una de las críticas descritas en el marco conceptual acerca de la elevada cantidad de compromisos que se plantean en las declaraciones conjuntas es un defecto que sí ha estado presente en el caso de esta relación birregional, lo cual puede ser interpretado como un excesivo planteamiento de propuestas que posteriormente no pueden cumplirse en su totalidad. Por tanto, más allá de su expresa representación en las declaraciones conjuntas, es importante crear mecanismos de seguimiento que verifiquen el cumplimiento de estos compromisos, generando transparencia en el proceso birregional para evitar que se erosione la credibilidad de las declaraciones emanadas de estos eventos y la legitimidad misma del proceso de cumbres. La falta de transparencia conduciría a un estado general de escepticismo hacia la diplomacia de cumbres que puede constituirse como un gran reto para esta práctica en el futuro.

Finalmente, y a modo de reflexión, cabe destacar que la emergencia de la crisis sanitaria por el Covid-19 aceleró la virtualización de gestiones que antes se circunscribían principalmente al contacto presencial. Así, el uso cada vez más difundido de las tecnologías de comunicación supone un avance importante para la transformación de las prácticas diplomáticas en el futuro. Sin embargo, es aún incierto si el nivel de comunicación que se logre en estas instancias virtuales podrá equipararse a lo que representa un encuentro personal entre los líderes políticos y si las ventajas de la diplomacia de cumbres se mantendrían aun cuando estos eventos se realicen de manera virtual. 


\section{Referencias bibliográficas}

Ayuso, Anna (2019). 20 años después. ¿Hacia dónde va la asociación estratégica? Pensamiento Propio, 49. 53-84.

Ayuso, Anna - Caballero, Sergio (2018). El interregionalismo de la Unión Europea con América Latina. Bogotá: Universidad Cooperativa de Colombia.

Ayuso, Anna - Gardini, Gian Luca (2018). EU-Latin American relations as a template for interregionalism. En: Mattheis, Frank - Litsegård, Andréas (eds.). Interregionalism across the Atlantic Space. London: Springer International Publishing. 115-130.

Ayuso, Anna - Gratius, Susanne (2016). América Latina y Europa: ¿Repetir o reinventar un ciclo? Pensamiento Propio, 44. 249-292.

Bouzas, Roberto (2019). EU-LAC Biregional Scenarios, EULAC Focus, Working Paper $\mathrm{N}^{\circ}$ WP6-D1-121.

Bretherton, Charlotte - Vogler, John (1999). The European Union as a global actor. New York: Routledge.

Cervantes, Rafael (2000). Una nueva asociación estratégica birregional: la Cumbre América Latina y el Caribe-Unión Europea. Revista Mexicana de Política Exterior, 61. 128-142.

Freres, Christian (2004). ¿De las declaraciones a la asociación birregional? Nueva Sociedad, 189. 110-124.

Freres, Christian - Sanahuja, José Antonio (2005). Study on Relations between the European Union and Latin America: New Strategies and Perspectives. Madrid: Instituto Complutense de Estudios Internacionales.

Gardini, Gian Luca - Malamud, Andrés (2016). Debunking Interregionalism: Concepts, Types and Critique - With a Transatlantic Focus. Atlantic Future, Working Paper No 38.

Grabendorff, Wolf (2004). La estrategia birregional y sus limitaciones en un mundo unipolar. Nueva Sociedad, 189. 97-109.

Grabendorff, Wolf (2003). América Latina y la Unión Europea: ¿Una asociación estratégica? En: Paz, Guadalupe - Roett, Riordan (comp.). América Latina en un entorno global en proceso de cambio. Buenos Aires: Nuevohacer Grupo Editor Latinoamericano. 187-205.

Malamud, Carlos (2010). Las relaciones entre la Unión Europea y América Latina en el siglo XXI: Entre el voluntarismo y la realidad. Plataforma Democrática, Working Paper $\mathrm{N}^{\circ} 6$. Melissen, Jan (2003). Summit diplomacy coming of age. Discussion papers in diplomacy, Working Paper $\mathrm{N}^{\circ} 86$.

Mori, Antonella (2018). EU and Latin America. A Stronger Partnership? Milan: Ledizioni LediPublishing.

Quevedo Flores, Jorge Alberto (2017). Perspectivas de la Asociación Estratégica Birregional eurolatinoamericana. En: Gutiérrez González, Alicia. - Arce Rodríguez, 
Diplomacia de cumbres a nivel interregional: un análisis desde la relación entre la Unión Europea y Latinoamérica

Lourdes Margarita (coords.). El futuro de la Unión Europea: Implicaciones para el sistema internacional. Guadalajara: Universidad de Guadalajara. 79-105.

Rojas Aravena, Francisco (2009). Diplomacia de Cumbres e integración regional. En: Jarque, Carlos - Ortíz, María Salvadora - Quenan, Carlos (eds.) América Latina y la Diplomacia de Cumbres. Madrid: Secretaría General Iberoamericana. 27-54.

Sanahuja, José Antonio (2004). Un diálogo estructurado y plural: La dimensión institucional de las relaciones Unión Europea-América Latina. Nueva Sociedad, 189. 80-96.

Yepe, Roberto (2011). La Diplomacia de Cumbres en América Latina y el Caribe. En: Bonilla, Adrián - Ortiz, Maria Salvadora (comp.) De Madrid a Santiago: Balances y perspectivas de las relaciones entre la Unión Europea y América Latina y el Caribe. San José: FLACSO. 245-250. 